\title{
Sensors, Signals, and Images in Medical Informatics: Progress and Evaluation
}

\section{Findings from the Yearbook 2008 Section on Sensors, Signals, and Imaging Informatics}

\author{
H. Müller, Managing Editor for the IMIA Yearbook Section on Sensors, Signals, and Imaging \\ Informatics \\ Medical Informatics, University Hospitals and University of Geneva \& \\ Business Information Systems, University of Applied Sciences Sierre, Switzerland
}

\section{Summary \\ Objectives: To summarize current research in the field of sensors, signals, and imaging in medicine and the impact of it in the medical informatics field through the selection of important and representative papers. \\ Methods: Survey of the 2007 biomedical literature in the area of sensors, signals, and imaging informatics. \\ Results: The review process of many candidate papers reflects the large variety of this research field. Four articles were finally selected with the help of the reviewers representing the important domains of brain-computer interfaces, brain shift correction, computer-aided interventions, and wearable sensors. \\ Conclusions: The four selected papers show the wide variety in medical informatics research concerning sensors, signals, and images. Imaging and signal research becomes increasingly broad and the number of techniques available and used in clinical practice is enormous and constantly increasing. The selected articles can only present a few highlights and many important topics had to be left out of this overview.}

\section{Keywords}

Medical informatics, International Medical Informatics Association, yearbook, sensors, signals, images

Geissbuhler A, KulikowskiC, editors. IMIA Yearbook of Medical Informatics 2008. Methods Inf Med 2008; 47 Supp 1:64-6

\section{Introduction}

The field of sensors, signals, and imaging in medical informatics comprises an very large domain of research. A selection of representative articles for this field is therefore difficult, the number of journals and the variety of topics being hard for anyone to know globally. The selection process for the articles goes in line with selections in previous years of the Yearbook of Medical Informatics [6,7] and particularly the sections on sensors, signals, and imaging informatics $[1,10]$.

Whereas the 2007 Yearbook contained several articles on sensors from tissue simulation [12], to wearable sensors [9] and brain-computer interfaces [8,11], the selection for 2008 contains more articles from the imaging domain $[3,4]$. Research in the fields of braincomputer interfaces [2] and wearable sensors [5] remain strong and active areas of research. Several articles in 2008 are from similar domains as the selection in 2007, though, showing the importance and high research quality in the field. Brain computer interfaces remain important [2] and will most likely be so for a few years to come. Wearable sensors [5] are equally still state of the art and the impact on clinical routine is likely to become increasingly visible.

\section{Best Paper Selection}

To select the papers for this Yearbook, twelve journals treating subjects concerning sensors, signals and images were analysed for the year 2007. The abstracts of all the articles were read and a selection was performed through an international peer-review process, taking into account the originality of the article, the impact of the topic, and the general interest in the field of medical informatics for the topic to avoid articles that are too specialist and of interest to only few persons. Besides the journals, a research in Pubmed was performed containing keywords frequent for imaging, signals, and sensors such as modalities (x-ray, MRI, etc.) or frequently used techniques.

In a first step around 100 articles were identified based on the titles and abstracts, and for the final review process this selection was reduced to 16 papers that were reviewed by three external reviewers, the two Yearbook editors and the section editor. The final selection based on the review scores represents the three application domains sensors [5], signals [2], and imaging $[3,4]$ as well as the various continents from North [4] and South America [3], to Europe [2], and Asia [5]. The first paper [2] describes the advances in brain-computer interfaces. Lovo et al 
Table 1 Best paper selection of articles for the IMIA Yearbook of Medical Informatics 2008 in the section 'Sensors, Signals, and Imaging Informatics'. The articles are listed in alphabetical order of the first author's surname.

\section{Section}

Sensors, Signals, and Imaging Informatics

- Friman 0, Volosyak I, Graser A. Multiple channel detection of steady-state visual evoked potentials for brain-computer interfaces. IEEE Trans Biomed Eng 2007;54(4):742-50.

- Lovo EE, Quintana JC, Puebla MC, Torrealba G, Santos JL, Lira IH, Tagle P. A novel, inexpensive method of image coregistration for applications in image-guided surgery using augmented reality. Neurosurgery 2007;60(4 Suppl 2):366-71;discussion 371-2.

- Reinertsen I, Lindseth F, Unsgaard G, Collins DL. Clinical validation of vessel-based registration for correction of brain-shift. Med Image Anal 2007;11(4):374-88.

- Zheng JW, Zhang ZB, Wu TH, Zhang Y. A wearable mobihealth care system supporting real-time diagnosis and alarm. Med Biol Eng Comput 2007;45(9):877-85.
[3] describe a method for using augmented reality as an aid for interventions (brain operations) concentrating on low cost techniques and open source software applicable in many contexts. Reinertsen et al. [4] also use brain-imaging techniques, this time using several modalities (MRI and ultrasound) to detect the displacement of cerebral structures (brain-shift) occurring when opening the skull for surgery. The last paper deals with sensors integrated into normal clothes to survey elderly and high-risk patients measuring vital parameters as well as detecting falls and sending the GPS coordinates of the persons via simple cell phone technologies.

Table 1 presents the articles finally selected for inclusion. Short summaries from all papers can be found on the following pages.

\section{Conclusions and Outlook}

Four articles representing main research directions in sensors, signals, and imaging informatics are presented in this section. The articles show the variety of research in this wide field and also the important impact that these techniques can have in clinical practice. Sensor, signal, and imaging informatics start to be evaluated on real world data sets and become increasingly used in clinical practice. Future Yearbooks will show whether all these techniques will become integrated with methods from other sections in this Yearbook such as bioinformatics, clinical data management, knowledge discovery, and decision support. Only such integration can lead to a real impact of this exciting research field.

\section{Acknowledgement}

Many thanks to Martina Hutter for her availability throughout the paper selection and article preparation process and to all the reviewers.

\section{References}

1. Westphal R. Sensors, Medical Image and Signal processing. Yearbook of Medical Informatics 2007, Methods Inf Med 2007;46 Suppl 1.

2. Friman O, Volosyak I, Graser A. Multiple channel detection of steady-state visual evoked potentials for brain-computer interfaces. IEEE Trans Biomed Eng 2007;Apr;54(4):742-50.

3. Lovo EE, Quintana JC, Puebla MC, Torrealba G, Santos JL, Lira IH, et al. A novel, inexpensive method of image coregistration for applications in image-guided surgery using augmented reality. Neurosurgery 2007;Apr;60(4 Suppl 2):36671; discussion 371-2.

4. Reinertsen I, Lindseth F, Unsgaard G, Collins DL. Clinical validation of vessel-based registration for correction of brain-shift. Med Image Anal 2007; June; 30 .

5. Zheng JW, Zhang ZB, Wu TH, Zhang Y. A wearable mobihealth care system supporting real-time diagnosis and alarm. Med Biol Eng Comput 2007; Sep;45(9):877-85.
6. Geissbuhler A,Haux R, Kulikowsky CA. Biomedical Informatics for sustainable health systems. Yearbook of Medical Informatics 2007, Methods Inf Med 2007;46 Suppl 1:6-8.

7. Haux R, Kulikowsky CA, editors. Assessing Information Technologies for Health. Yearbook of Medical Informatics 2006. Methods Inf Med 2006; 45 Suppl 1 .

8. Kim HK, Biggs SJ, Schloerb DW, Carmena JM, Lebedev MA, Nicolelis MA, et al. Continuous shared control for stabilizing reaching and grasping with brainmachine interfaces. IEEE Trans Biomed Eng 2006 Jun;53(6):1164-73.

9. Parkka J, Ermes M, Korpipaa P, Mantyjarvi J, Peltola $\mathrm{J}$, Korhonen I. Activity classification using realistic data from wearable sensors. IEEE Trans Inf Technol Biomed 2006 Jan;10(1):119-28.

10. Westphal R, Winkelmann S. Sensor, Signal and Imaging Informatics - State of the Art and Current Topics. Methods Inf Med 2006:68-71.

11. Moore Jackson MM, Mason SG, Birch GE. Analyzing trends in brain interface technology: a method to compare studies. Ann Biomed Eng 2006;34(5):859-78.

12. DiMaio SP, Salcudean SE. Needle steering and motion planning in soft tissues. IEEE Trans Biomed Eng 2005 Jun;52(6);965-74.

\section{Correspondence to:}

Prof. Dr. Henning Müller

University \& Hospitals of Geneva

Medical Informatics and

University of Applied Sciences

Business Information Systems

TecnoARK 3

3960 Sierre, Switzerland

Tel: +41 276069012

Fax: +41276069000

E-mail: henning.mueller@hevs.ch

\author{
Friman 0, Volosyak I, Graser A \\ Multiple channel detection of steady-state \\ visual evoked potentials for brain-computer \\ interfaces
} * The complete papers can be accessed in the Yearbook's
full electronic version, provided that permission has
been granted by the copyright holder(s) 


\section{IEEE Trans Biomed Eng 2007;Apr; 54(4)::742-50}

Brain-computer interfaces have been a hot topic in signal processing over the past five years and first systems start having very good results for simple tasks. Friman et al. propose a new method for brain computer interfaces with several EEG (electroencephalogram) recordings. A challenge is to cancel out the noise, reach a high detection rate, and at the same time have a very quick processing speed. An evaluation with ten subjects was performed to validate the approaches. Several approaches were implemented and evaluated, with the best leading to a high detection rate of $84 \%$, showing the progress in brain computer interfaces over the past years.

\section{Lovo EE, Quintana JC, Puebla MC, Torrealba} G, Santos JL, Lira IH, Tagle P

A novel, inexpensive method of image coregistration for applications in imageguided surgery using augmented reality Neurosurgery 2007;Apr;60(4 Suppl 2):36671;discussion 371-2

Lovo et al. present an article on imageguided surgery using virtual reality approaches. The particularity of their approach is the use of open source software and simple digital cameras instead of the often extremely expensive virtual reality equipment used in many industrialised countries. 3D images from MRI scans are co-registered with intra-operative ultrasound and simple photographs to help the surgeons visualise the information. Concrete experiments were performed and evaluated on eight patients and directly in clinical practice. The results show that the technique is well adapted and can be used in a large variety of settings, without the use of important financial resources.

\section{Reinertsen I, Lindseth F, Unsgaard G, Collins DL Validation of vessel-based registration for correction of brain-shift}

\section{Med Image Anal 2007;11(4):374-88}

Brain shift after opening the skull for an operation is a well-known and often studied problem potentially leading to serious errors during the operation due to shifted tissue. Reinertsen et al. present a new approach to detecting and correcting brain shift with using preoperative MRI, and then simple ultrasound during the operation to identify potentially shifted brain parts. In both imaging modalities vessels are segmented and then matched for better registration of the modalities during the operation. Validation was first per- formed with simulated datasets and then a phantom study very close to clinical reality showing very good mapping results.

\section{Zheng JW, Zhang ZB, Wu TH, Zhang Y \\ A wearable mobihealth care system supporting real-time diagnosis and alarm \\ Med Biol Eng Comput 2007; Sep; 45(9):877-85}

Zheng et al. describe a wearable monitoring system acquiring ECG data and respiratory parameters from a patient. The acquisition is directly integrated in the fabric of the clothes. Communication of the fabric integrated system is enabled via wireless short-range transmissions towards a processing unit. The processing unit itself is connected via cell phone technology to a medical institution. Further sensors of the system include an acceleration sensor to detect falls and long periods without any motion, with the goal to quickly detect abnormal situations. A GPS unit allows the clinicians to locate the person and give either advice via phone or send an ambulance in case of an emergency. Application area of the system is mainly the constant supervision of elderly and high-risk patients with vascular diseases. 